\title{
Fusion around the barrier for ${ }^{7} \mathrm{Li}+{ }^{12} \mathrm{C}$
}

\author{
A MUKHERJEE ${ }^{1}$, M DASGUPTA ${ }^{1}$, D J HINDE ${ }^{1}$, C R MORTON ${ }^{1}$, A C BERRIMAN ${ }^{1}$,

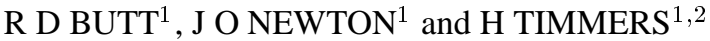 \\ ${ }^{1}$ Department of Nuclear Physics, ${ }^{2}$ Department of Electronic Materials Engineering, Research \\ School of Physical Sciences and Engineering, Australian National University, Canberra, ACT 0200, \\ Australia
}

\begin{abstract}
Fusion cross-sections for the ${ }^{7} \mathrm{Li}+{ }^{12} \mathrm{C}$ reaction have been measured at energies above the Coulomb barrier by the direct detection of evaporation residues. The heavy evaporation residues with energies below $3 \mathrm{MeV}$ could not be separated out from the $\alpha$-particles in the spectrum and hence their contribution was estimated using statistical model calculations. The present work indicates that suppression of fusion cross-sections due to the breakup of ${ }^{7} \mathrm{Li}$ may not be significant for ${ }^{7} \mathrm{Li}+{ }^{12} \mathrm{C}$ reaction at energies around the barrier.
\end{abstract}

Keywords. Fusion; breakup; light ion fusion.

PACS No. 25.70.Jj

\section{Introduction}

Considerable interest has been devoted in recent years towards understanding the mechanism of fusion reactions involving weakly bound stable nuclei, like ${ }^{6} \mathrm{Li},{ }^{7} \mathrm{Li}$ and ${ }^{9} \mathrm{Be}$ [1-13]. When one of the colliding nuclei is loosely bound, owing to its low binding energy, it may break up in the field of the other nucleus, and can thereby influence the fusion process. However, the effect of this breakup process on the fusion cross-sections is still controversial $[9,11,13]$, with some authors predicting an enhancement due to the coupling of the breakup channel(s) to the fusion process, while others predicting a suppression of fusion cross-sections due to the break up of the weakly bound nucleus before fusing to form a compound nucleus. However, as far as the experimental fusion cross-sections for light heavy-ion reactions, like ${ }^{6,7} \mathrm{Li}+{ }^{12,13} \mathrm{C}$ and ${ }^{6,7} \mathrm{Li}+{ }^{16} \mathrm{O}$, are concerned, there exists a considerable discrepancy between different measurements carried out at energies around the barrier. Some authors observe fusion cross-sections for these systems to be close to the total reaction cross-sections at energies around the barrier [4-6,8,10], while others observe a strong limitation of fusion cross-sections [1-3,7] at low energies. Consequently, there exists an experimental controversy regarding the effect of the breakup of these loosely bound nuclei on the fusion cross-sections of light heavy-ion systems.

In order to resolve this discrepancy, fusion cross-sections measurements were carried out at the Australian National University, for the ${ }^{7} \mathrm{Li}+{ }^{12} \mathrm{C}$ reaction at energies from about two to five times the Coulomb barrier energy. 


\section{Experiment}

The experiments were performed with ${ }^{7} \mathrm{Li}$ beams in the energy range of $13.0-30.0 \mathrm{MeV}$, using the 14UD tandem accelerator at the Australian National University. The target employed was ${ }^{12} \mathrm{C}$ of thickness $15 \mu \mathrm{g} / \mathrm{cm}^{2}$ with a $1 \mu \mathrm{g} / \mathrm{cm}^{2}$ Au flash. Two monitor detectors were placed at angles of $\pm 10^{\circ}$ with respect to the beam direction to measure the elastically scattered beam particles for normalization. Ions of ${ }^{16} \mathrm{O}$ at beam energies of 15 and $19 \mathrm{MeV}$ were elastically scattered from the target to calibrate the product of the target thickness and detector solid angle. The fusion cross-sections were measured by detecting the heavy evaporation residues (ERs) resulting from compound nucleus formation and subsequent particle evaporation. The nuclear charge $Z$ of the individual reaction products was identified by using a $\Delta E-E$ telescope [14] consisting of a gas-ionization detector mounted on a movable arm inside a scattering chamber of diameter $2 \mathrm{~m}$. The detector has a $70 \mu \mathrm{g} / \mathrm{cm}^{2}$ thick mylar window. Propane gas was passed through the detector at selected pressures between 50 and 100 mbar, depending on beam energy, chosen so as to stop all the ERs well within the detector.

A typical two dimensional $\Delta E-E$ spectrum for the ${ }^{7} \mathrm{Li}+{ }^{12} \mathrm{C}$ reaction is shown in figure 1, where $E$ is the total energy of the ERs. Fusion products were defined as those nuclei heavier than $\mathrm{Li}$. In order to eliminate the contribution from $\alpha$-particles an energy cut of $3 \mathrm{MeV}$ had to be applied while estimating the contribution of the ERs towards fusion cross-sections. The reliablility of the present setup was established by measuring the fusion cross-sections for the ${ }^{12} \mathrm{C}+{ }^{12} \mathrm{C}$ reaction at the c.m. energies of 15 and $20 \mathrm{MeV}$. This reaction was chosen as the reference reaction, as this is a well-studied system. Fusion cross-sections have been measured by all the three different techniques, the $\gamma$-ray method [15], the TOF method [16] and the $\Delta E-E$ method [17], and the results are in reasonably good agreement with each other.

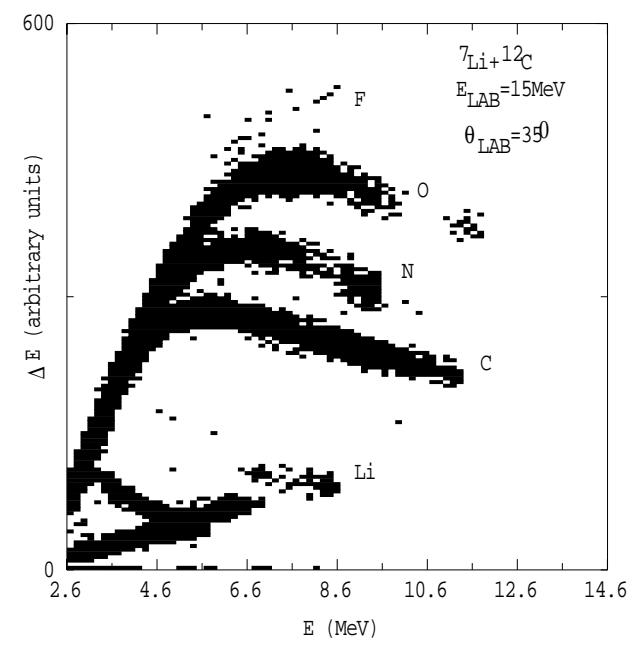

Figure 1. A typical two-dimensional $\Delta E-E$ spectrum for the ${ }^{7} \mathrm{Li}+{ }^{12} \mathrm{C}$ reaction. 


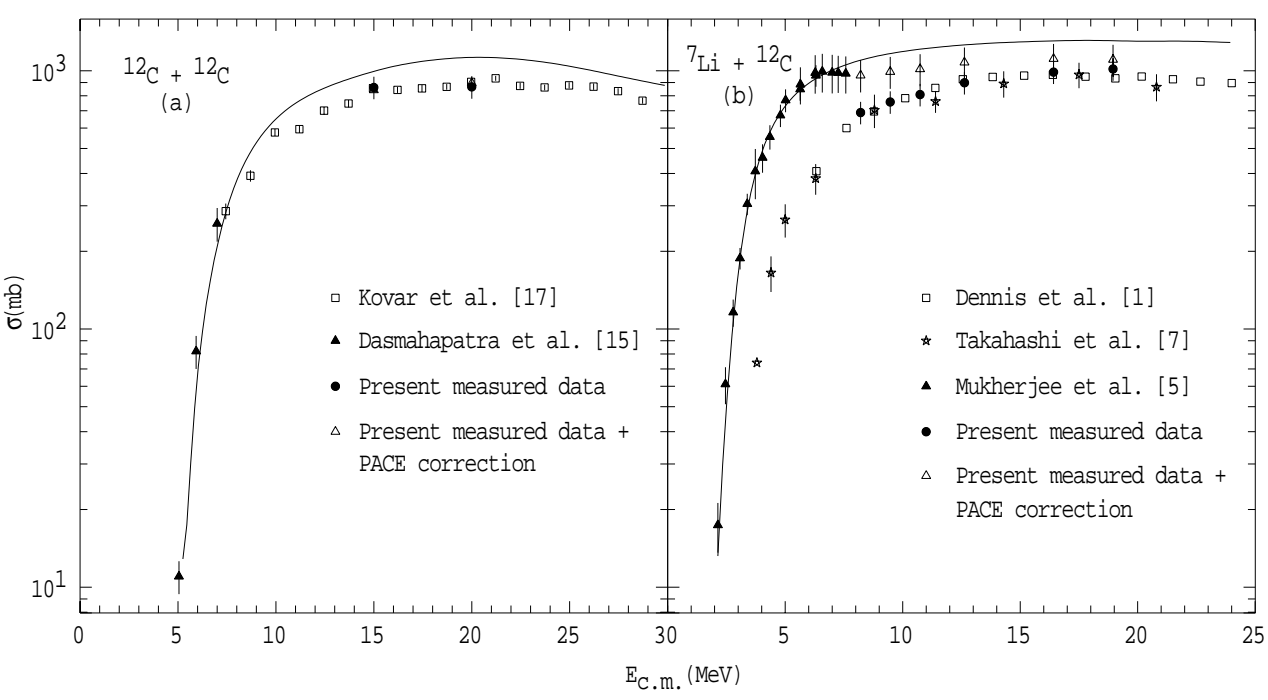

Figure 2. Fusion cross-sections for (a) ${ }^{12} \mathrm{C}+{ }^{12} \mathrm{C}$ and (b) ${ }^{7} \mathrm{Li}+{ }^{12} \mathrm{C}$ reactions. The solid curves are the optical model calculations obtained with parameters from (a) ref. [18] and (b) ref. [19].

Evaporation residues were measured at laboratory angles of $16^{\circ}, 20^{\circ}, 25^{\circ}, 30^{\circ}$ and $35^{\circ}$, and these matched well with the previous measurements [1,17], for both the systems. Therefore, taking the angular distributions at more forward angles to be the same as measured previously, total fusion cross-sections were then obtained.

Figure 2 shows the fusion cross-sections obtained in the present work for the ${ }^{12} \mathrm{C}+{ }^{12} \mathrm{C}$ and ${ }^{7} \mathrm{Li}+{ }^{12} \mathrm{C}$ reactions. The very good agreement obtained for the ${ }^{12} \mathrm{C}+{ }^{12} \mathrm{C}$ system establishes the reliability of the present setup. The solid curves in figure 2 represent the total reaction cross-sections obtained using the optical model calculations performed with parameters taken from ref. [18] for ${ }^{12} \mathrm{C}+{ }^{12} \mathrm{C}$ system and ref. [19] for ${ }^{7} \mathrm{Li}+{ }^{12} \mathrm{C}$ system.

\section{Discussion}

From figure $2 \mathrm{~b}$ it can be seen that for the ${ }^{7} \mathrm{Li}+{ }^{12} \mathrm{C}$ system, the fusion cross-sections of the present work (shown by the symbol $\bullet$ ) agree with the cross-sections of the earlier works [1,7] obtained by the direct detection of ERs; the present measurements however do not agree with those of ref. [5] obtained using the $\gamma$-ray technique. However, it has already been mentioned in $\S 2$ that a low energy cut-off at $3 \mathrm{MeV}$ was applied in the present work. This means that the contribution of the ERs of energies below $3 \mathrm{MeV}$ has not been included in determining the fusion cross-sections as shown.

An estimate of the amount of these missing cross-sections was obtained with the help of the statistical mode code, PACE. The reliability of using the PACE calculations was checked by comparing the calculated energy distribution and the mean energy of the ERs with those measured, for both the systems. This contribution in the ${ }^{12} \mathrm{C}+{ }^{12} \mathrm{C}$ reaction 
is below $1 \%$ at c.m. energies of 15 and $20 \mathrm{MeV}$, and it remains below $10 \%$ even down to $E_{\text {c.m. }}=7.5 \mathrm{MeV}$, which is the lowest energy of measurement in ref. [17]. However, for the ${ }^{7} \mathrm{Li}+{ }^{12} \mathrm{C}$ reaction, PACE calculations predict the contribution of evaporation residues below $3 \mathrm{MeV}$ to be $\sim 25 \%$ at $E_{\text {c.m. }}=9.5 \mathrm{MeV}$, and $\sim 70 \%$ at $E_{\text {c.m. }}=3.8 \mathrm{MeV}$. The present measured cross-sections after being corrected for the missing contributions below $3 \mathrm{MeV}$, as predicted by PACE, are shown in figure 2 by the symbol $\Delta$ for both the reactions. The error bars for these corrected fusion cross-sections include a $10 \%$ error for PACE calculations, in addition to the experimental errors shown for the measured cross sections (symbol $\bullet$ ). It can be seen from figure $2 b$ that these corrected fusion cross-sections for ${ }^{7} \mathrm{Li}$ $+{ }^{12} \mathrm{C}$ match well with the $\gamma$-ray measurements. Therefore, the cross-sections measured in this work, even though seeming to agree with those of Dennis et al [1], are actually under-estimated by the amount of the missing contribution from the ERs of energies below $3 \mathrm{MeV}$. The actual amount of this contribution may differ somewhat from the PACE calculations, but the true fusion cross-sections are bound to be higher than those measured in the present work. It is worthwhile noting in this context, that unlike the direct detections technique, the $\gamma$-ray method is not affected by the low energy of the ERs, as in this method one detects the $\gamma$-rays emitted by the ERs.

Therefore, from the present work, we see that the large suppression of fusion crosssections in the ${ }^{7} \mathrm{Li}+{ }^{12} \mathrm{C}$ reaction at lower energies as claimed in the previous work [7] may be very significantly affected by the low energy cut-off in their detection system. Further measurements to quantify this suggestion are in progress.

\section{References}

[1] L C Dennis et al, Phys. Rev. C26, 981 (1982)

[2] J F Mateja et al, Phys. Rev. C30, 134 (1984)

[3] J F Mateja et al, Phys. Rev. C33, 1649 (1986)

[4] C J S Scholz et al, Z. Phys. A325, 203 (1986)

[5] A Mukherjee et al, Nucl. Phys. A596, 299(1996)

[6] A Mukherjee and B Dasmahapatra, Nucl. Phys. A614, 238 (1997)

[7] J Takahashi et al, Phys. Rev. Lett. 78, 30 (1997)

[8] A Mukherjee et al, Nucl. Phys. A635, 205(1998)

[9] M Dasgupta et al, Phys. Rev. Lett 82, 1395 (1999) and references therein

[10] A Mukherjee et al, Nucl. Phys. A645, 13 (1999)

[11] M Dasgupta et al, Proc. Int. Workshop on Fusion Dynamics at the Extremes (Dubna, 2000) to appear in World Scientific

[12] S B Moraes et al, Phys. Rev. C61, 064608 (2000)

[13] K Hagino et al, Phys. Rev. C61, 037602 (2000)

[14] H Timmers et al, Nucl. Instr. and Meth. B161-163, 19 (2000)

[15] B Dasmahapatra et al, Nucl. Phys. A384, 257 (1982)

[16] B Heusch et al, Phys. Rev. C23, 1527 (1981)

[17] D G Kovar et al, Phys. Rev. C20, 1305 (1979)

[18] H Reeves, Astrophys. J. 146, 447 (1966)

[19] J E Poling et al, Phys. Rev. C13, 648 (1976) 